\title{
Nachruf auf Prof. Dr. Franz FERSCHL
}

\section{Ein persönlicher Nachruf auf Franz Ferschl}

\author{
Gustav Feichtinger, Wien
}

Es war im Studienjahr 1959/60 als ich FF kennen lernte. Der Seelsorger meines Studentenheims schickte mich zu ihm, um einen philosophischen Seminarvortrag über Bochensky vorzubereiten. "Ferschl ist unheimlich klug, Du kannst von ihm viel profitieren" meinte Père Michel - und das hat sich dann auf ungeahnte Weise bewahrheitet.

Zunächst ist mir die Art aufgefallen, mit der FF Sachverhalte erklärte: sein enorm breit gestreutes Tatsachenwissen, verwirkt mit seinem speziellen "Erklärungsstil". In diesem spiegelte sich seine weltanschauliche Position wider, die zwar auf tiefer Religiosität beruhte, aber auch scheinbar feste Tatsachen stets kritisch hinterfragte. Jeder der ihn näher kannte weiß, was ich meine.

Bald danach kreuzten sich unsere Wege abermals. Wilfried Nöbauer schrieb 1958 gemeinsam mit FF eine Arbeit über Polynomgruppen und regte eine Weiterführung des Themas im Rahmen meiner Dissertation an. Nun hatte ich Gelegenheit, Ferschls Talent als Algebraiker kennenzulernen. Die Gruppentheorie ist bis an sein Lebensende eines seiner bevorzugten Hobbies geblieben.

Ferschls eigentliche akademische Karriere begann mit seiner Habilitation über Stochastik. Tagsüber war er in der Bundeskammer für Gewerbliche Wirtschaft als Referent für Statistik tätig. In der Nacht schrieb er an seinem Buch über Warteschlangentheorie, das dann 1964 unter dem Titel "Zufallsabhängige Wirtschaftsprozesse" erschien. Dieses Werk verschaffte ihm alsbald einen Ruf an die Rechts- und Staatswissenschaftliche Fakultät der Rheinischen Friedrich-Wilhelms-Universität in Bonn. Ich hatte die Chance, ihn - neben Klaus Steiner - als Assistent dorthin zu begleiten.

Die Wirtschaftswissenschaften waren in Bonn bis zur Berufung von Wilhelm Krelle "verbal" ausgerichtet. Zu Beginn der Sechziger Jahre "drehte" Krelle die Fakultät binnen kurzem. Das "Bonner Dreigestirn” Krelle-Albach-Beckmann favorisierte mathematische Methoden in der ökonomischen Forschung, was sich auch in der Ausbildung niederschlug. Rasch wurde Bonn ein Zentrum für quantitative Wirtschaftsforschung, wofür natürlich statistische Methoden wichtig waren.

Die Vorlesungen aus Statistik am Bonner Institut für Gesellschafts- und Wirtschaftswissenschaften lagen vor $1965 \mathrm{im}$ argen. Viel mehr als Mittelwerte, Varianzen und Beispiele für Normalverteilungen wurden nicht präsentiert. Es fehlte ein solides Angebot statistischer und wahrscheinlichkeitstheoretischer Methoden, die zum Verständnis von VWL, BWL und OR nötig sind. FF startete ab dem Studienjahr 1965/66 ein ehrgeiziges Programm. Neben einer zwei-semestrigen Methodenlehre für Statistik las er Stichprobentheorie, Regressionsmodelle, statistische Testtheorie, Einführung in stochastische Prozesse u.a.m.

Vor mir liegt ein Ferschl-Manuskript aus der damaligen Zeit. In gestochen scharfer Schrift präsentiert er Modelle der Erneuerungs- und Warteschlangentheorie in charakteristischer Art. Ohne Verwendung von Maßtheorie versucht er Lesern und Hörern schwierige Probleme auf verständliche Weise darzubieten - mit Erfolg, wie die Reaktionen vieler Mitarbeiter und Studenten zeigten. Eine Reihe von ihnen, die in der Statistischen Abteilung als Tutoren, Hilfskräfte bzw. Assistenten begonnen haben, sind (oder waren) als Professoren einschlägig tätig. 
FF beschäftigte sich zu dieser Zeit mit "Assemblageprozessen", die an der Schnittstelle von Erneuerungs- und Bedienungstheorie angesiedelt sind. Seine Untersuchungen sind dem Operations Research zuzurechnen, bzw. der Unternehmensforschung, wie das Gebiet damals genannt wurde. Er war damals auch Vizepräsident der Deutschen Gesellschaft für Unternehmensforschung (DGU). Die umfangreichste OR-Bibliothek im deutschen Sprachraum, die Literaturstelle für Unternehmensforschung, war seiner Leitung anvertraut.

Persönlich sind Ferschls Bonner Jahre durch die Breite seiner Aktivitäten gekennzeichnet, die aber keineswegs auf Kosten der Tiefe gingen. Mit der ihm eigenen Akribie widmete es sich der statistischen Methodenlehre, Wahrscheinlichkeitstheorie, dem Operations Research, der Wirtschaftstheorie und, last but not least, philosophischen Fragestellungen. Man konnte mit allen Problemen und Fragen zu ihm kommen und wurde nie von seinen Antworten enttäuscht. Die Palette seiner Lehrveranstaltungen erstreckte sich von der Wirtschaftsstatistik - die er ja als ehemaliger Statistiker an der Wirtschaftskammer aus dem ff (sic!) beherrschte - bis zu Zufallsprozessen und Entscheidungstheorie. In guter Erinnerung sind mir seine Seminare über Stichprobentheorie und Markoffketten.

Letztere leiten zu einem von ihm auch geschätzten Gebiet über, nämlich der Bevölkerungsmathematik, d.h. der formalen Demographie. Ferschl hat schon vor vierzig Jahren, wohl als erster im deutschen Sprachraum, deren Bedeutung erkannt. Daneben war FF in der Umbruchszeit der Endsechziger Dekan der Rechts- und Staatswissenschaftlichen Fakultät der Universität Bonn. Als Assistentenvertreter habe ich seine Bemühungen um Konfliktbewältigung hautnah miterlebt, etwa bei Dekanatsbesetzungen durch den Kommunistischen Studentenverband.

Seminarwanderungen waren in Bonn nichts Außergewöhnliches. FF hat seine Mitarbeiter auch an Wochenenden mit auf den Rheinhöhenweg und in die Eifel genommen.

FF war ein allseits beliebter Lehrer und Kollege. Sein Kontakt mit den Studenten war ungemein intensiv; seine Diskussionsfreudigkeit legendär. In diese Zeit fällt auch FFs Begegnung mit Inge Ferschl, geb. Lünen, die ihm dann sein ganzes Leben treu zur Seite gestanden ist.

Ich möchte diesen "persönlichen Nachruf" mit drei kurzen Erinnerungen an meinen Freund, Chef und akademischen Lehrer schließen:

- Unter "Anführung" von Prof. Adam durften wir Assistenten zum Antrittsbesuch Ferschls bei der Familie Lünen von Bonn nach Wattenscheid ins Ruhrgebiet mitfahren und sozusagen gemeinsam Verlobung feiern.

- Zu Semesterende waren die Wochenenden zur Klausurkorrektur reserviert. Samstag vormittag wurde die Statistikklausur - in vier Gruppen um Schwindeleien vorzubeugen - abgehalten, und bis Montag früh mußten die Verbesserungen vorliegen natürlich alles unter Chefaufsicht.

- Bei der Durchmusterung des Magazins der Institutsbibliothek am Beginn unserer Bonner Zeit hat Ferschl jedes der vielen dort lagernden Statistikbücher vom Staub abgeklopft, durchgeblättert und dabei uns Assistenten über den Inhalt erzählt.

So ist mir Franz Ferschl in Erinnerung geblieben.

Wien, im August 2007

Gustav Feichtinger 


\title{
Nachruf auf Franz Ferschl - Franz Ferschls Bonner Zeit
}

\author{
Klaus-Peter Kistner, Bielefeld
}

Ehe Franz Ferschl den Ruf auf den neu geschaffenen Lehrstuhl für Statistik an der Rechts- und Staatswissenschaftlichen Fakultät der Rheinischen Friedrich-Wilhelms-Universität in Bonn annahm, war die Statistik-Ausbildung im Diplom-Studiengang für Volkswirte in einem desolaten Zustand: Es wurde lediglich eine zweistündige Grundlagenveranstaltung der Deskriptiven Statistik angeboten. Die Dozenten waren entweder Privatdozenten der Rechts- und Staatswissenschaftlichen Fakultät mit volkswirtschaftlichem Hintergrund oder außerplanmäßige Professoren der Mathematisch-Naturwissenschaftlichen Fakultät. Trotz der sich abzeichnenden quantitativen Ausrichtung der volkswirtschaftlichen Ausbildung wurden weiterführende Veranstaltungen zur Statistik nicht angeboten. Für das gemäß Studienordnung zugelassenen Wahlfach Statistik waren die Studenten entweder auf den Besuch einschlägiger Vorlesungen an der Universität Köln angewiesen, oder sie mussten zumindest zeitweilig an andere Universitäten wechseln.

Diese Situation änderte sich schlagartige mit der Übernahme des Lehrstuhls durch Franz Ferschl im Herbst 1965. Er erschien mit zwei Akademischen Räten aus Wien, einer mit mathematischer, einer mit wirtschaftswissenschaftlichem Hintergrund - ein Physiker gesellte sich bald dazu - und zeigte den Bonner Studenten der Volkswirtschaftslehre nicht nur, was Statistik war, sondern mischte auch kräftig in der Ausbildung der Unternehmensforschung und im Operations Research mit. Als Grundlage der StatistikAusbildung entwickelte er einen zweisemestrigen Vorlesungs-Zyklus, in dem die deskriptive und die schließende Statistik in anspruchsvoller, aber trotzdem der Ausbildung von Diplom-Volkswirten angemessener Weise dargestellt wurde. An diese schlossen sich weiterführende Veranstaltungen im Rahmen des Wahlfachs Statistik und Ökonometrie an, in denen Franz Ferschl ebenfalls in hervorragender Weise die Gratwanderung zwischen mathematischer Rigidität und Verständlichkeit und Anwendungsbezug für künftige Wirtschaftswissenschaftler meisterte. Auch seine Vorlesungen im Bereich der Unternehmensforschung waren vorbildlich in Hinblick auf Genauigkeit und Verständlichkeit. Mir sind insbesondere seine Vorlesungen über Markov-Ketten und über Entscheidungstheorie in Erinnerung. Zusammen mit seiner Habilitationsschrift über "Zufallsabhängige Wirtschaftsprozesse" waren sie mir ein zentraler Zugang zu der mich während meiner gesamten wissenschaftlichen Laufbahn beschäftigenden Stochastik. Allerdings ist anzumerken, dass sich hartnäckig das Gerücht hielt, die Hauptschwierigkeit für die Hörer seinen Vorlesungen nicht die Mathematik, sondern sein leicht österreicherischer Tonfall gewesen sein.

Franz Ferschl hat sich in außergewöhnlicher Weise in die damalige Lehre und Forschung der Bonner Volks- und Betriebswirte integriert. Er war nicht nur inhaltlich eine unverzichtbare Ergänzung des "Bonner Dreigestirn" - Wilhelm Krelle, Martin Beckmann und Horst Albach - auch die persönlichen Beziehung zwischen ihm und den maßgeblichen Bonner Kollegen war vorbildlich. Es ist auch ihm zu verdanken, dass der Fachbereich nach außen hin einen geschlossenen und äußerst fruchtbaren homogenen Eindruck vermitteln konnte. Nicht zuletzt ihm ist es zu verdanken, dass die Bonner Fakultät einen über die Grenzen Deutschlands reichenden Ruf erwerben konnte. Dieses wird insbesondere durch die Tatsache, dass der Sonderforschungsbereich "Ökonometrie und Un- 
ternehmensforschung" als einer der ersten eingeworben werden konnte. Auch die damaligen Studenten und Mitarbeiter der Fakultät waren von dieser wissenschaftlichen und persönlichen Geschlossenheit beeindruckt und profitierten von der homogenen wissenschaftlichen Ausbildung während Franz Ferschls Bonner Zeit.

Von dem wissenschaftlichen Ruf, den sich Franz Ferschl während dieser Zeit erwerben konnte, zeugt nicht zuletzt die Tatsache, dass er zum Vorsitzen der Deutschen Gesellschaft für Unternehmensforschung, dem Kern der späteren Gesellschaft für Operations Research gewählt wurde.

Mit Ferschls Zeit in Bonn fiel der Umbruch der akademischen Landschaft in Deutschland zusammen: $\mathrm{Zu}$ seine Antrittsvorlesung - dem Anlass der Aufnahme eines Statistikers in die Rechts- und Staatswissenschaftliche Fakultät entsprechen sprach er "Zur Problematik des Bays'schen Theorems am Beispiel der Hexenprozesse" - erschienen Rektor und Dekan im Ornat, er und seine Kollegen im Talar. Sein Dekanat stand wenige Jahre später unter dem Zeichen der Studentenrevolte. Es ist bewundernswert, mit welcher Ruhe und Gelassenheit er dieses Amt in schweren Jahren verwaltete. Allen Teilnehmern an den für diese Zeit kennzeichnenden Veranstaltungen bleibt sein meisterhaft eingesetzter scholastischer Diskurs mit "Studentenvertretern", akademischen Argumentationen nicht immer zugänglichen waren, in Erinnerung. Allein er hätte die Frage beantworten können, ob die Diffamierungen, die auch ihn in seinem Amt als Hochschullehrer und Dekan trafen, letztlich maßgeblich für seinen Entschluss, Bonn zu verlassen, waren oder ob der Reiz, den die Universität Wien für einen österreichschen Wissenschaftler ausübt, ihn dazu verleitet hatte, einen Ruf an diese Universität anzunehmen. Für alle Mitglieder der Bonner Fakultät war seine Entscheidung auf jeden Fall nur schwer zu verstehen, zumal er noch während Bonner Zeit eine Wahlbonnerin geheiratet hat.

Für seinen Einsatz für seine Mitarbeiter und den wissenschaftlichen Nachwuchs zeugt die Tatsache, dass er während der relativ kurzen Zeit in Bonn zwei seiner Mitarbeiter, Christoph Schneeweiß und Gustav Feichtinger habilitierte; deren Qualifikation wird durch die Tatsache unterstrichen, dass sie innerhalb sehr kurzer Zeit auf Ordentliche Professuren in Deutschland bzw. in Österreich berufen wurden.

Auch ich bin durch Franz Ferschl in meiner wissenschaftlichen Laufbahn gefördert und bestärkt worden. Schon bei meiner Diplom-Prüfung hat er hospitiert und wahrscheinlich durch seine Anwesenheit zu einem guten Ergebnis maßgeblich beigetragen. Mein Habilitationsprojekt über einen betriebswirtschaftlichen Aspekt der Warteschlangentheorie hat er mitbetreut und beeinflusst. Obwohl er in seinem Gutachten die Annahme als Habilitationsschrift empfohlen hat, hat er mir eine Fülle von Änderungswünschen zukommen lassen. Auch wenn ich mich nach der Berufung auf einen Lehrstuhl für Betriebswirtschaftslehre nicht in dem von ihm möglicherweise gewünschten Umfang mit Anwendungen stochastischer Prozesse befassen konnte, hat er mich immer wieder dazu ermuntert, auf diesem Gebiet weiter zu arbeiten. Runde Geburtstage gaben mir aber immer wieder einen Anstoß, mich wieder mit Warteschlangen zu befassen.

Auch wenn ich nicht zum engsten Kreis seiner Schüler gehört habe, so bin ich Franz Ferschl doch zu großem Dank verpflichtet und werde seinen Einfluss auf meine wissenschaftliche Entwicklung immer zu schätzen wissen.

Bielefeld, im August 2007

Klaus-Peter Kistner 


\title{
F. Ferschls Wiener Jahre, eine persönliche Sicht
}

\author{
Wolfgang Polasek, Wien
}

Franz Ferschl wurde als 3. Statistikprofessor neben S. Sagoroff and G. Bruckmann an das Institut für Statistik an der Universität Wien berufen. Damals war das Institut im Erdgeschoss des Neuen Institutsgebäudes (NIG) in unmittelbarer Nähe des Rechenzentrums (das gerade eine neue IBM360 beherbergte). Dort war es auch, wo ich F. Ferschl zum ersten Mal sah, als er gerade nach Wien kam und das Institut besuchte. Da es zu wenig Platz gab, erhielt der neue Professor von der Uni Räumlichkeiten in einem Gründerzeithaus am Rooseveltplatz. Nur die Bibliothek war im NIG und wurde auf Wunsch von Franz Ferschl sogleich von einer Seriennummer-Aufstellung in ein Sachgebietsystem umgestellt. Das war damals auch mein erster Job am Institut, das alle Institutsanghörige in den Sommerferien neben Staub auch einige Diskussionen bescherte. Als weitere Aufbauarbeit am Institut war im ersten Jahr die Herausgabe der Skripten Statistik 1 und 2 geplant (und die in ein Buchprojekt münden sollte ${ }^{1}$ ), wobei parallel dazu die Aufgaben- und Formelsammlungen für die Übungen erstellt wurden, welche F. Ferschl ein besonderes Anliegen waren. In der Oberstufe las F. Ferschl die Ökonometrie, die neben OR ein Pflichtwahlfach für die Statistikstudenten war. Dann gab es als intellektuelle Höhepunkte die Statistik-Seminare in jedem Semester, die F. Ferschl als erstes den Grundlagenthemen widmete: War doch gerade das mehrbändige Werk W. Stegmüller (Universität München) über Wissenschaftstheorie und statistische Begründung herausgekommen und diskutierte die objektiven und subjektiven Grundlagen von Empirie und Statistik.

Der Aufbau des neuen Magisterstudiums für Sozial- und Wirtschaftsstatistik war Anfang der 70er Jahre die wichtigste Aufgabe des Instituts für Statistik. Nach dem neuen SoWi-Studiengesetz waren die Studienrichtungen von Volkswirtschaft, Soziologie und Statistik in der Unterstufe (bis zum 1. Diplom) weitgehend identisch aufgebaut. Dies machte sich besonders in den Einführungsvorlesungen für Statistik und Mathematik bemerkbar, die vom Institut betreut wurden. Die Vorlesungen waren im Hauptgebäude (HS28) und am Weg dahin bekam man in jenen Zeiten jeden Tag einige Flugblätter in die Hand gedrückt. F. Ferschl war ein aufmerksamer Leser und Sammler dieser Flugzettel. Er war damit ein belesener Kenner der studentischen Strömungen an der Uni Wien, und er hatte $\mathrm{Spa}$ mit dem gärenden Kern der studentischen 70er Jahre vor, während und nach der Vorlesung zu diskutieren. Denn: die 68er Generation saß in den Hörsälen, und in Österreich kam damals dazu, dass diese Generation parallel zur Kreisky-Ära ausgebildet wurden - einer "neuen Zeit", wo seit der Nationalratswahl 1970 die Vorstellung aufkam, Österreich als eine "offene Gesellschaft" nach skandinavischem Vorbild umzubilden. Die Sozial- und Wirtschaftswissenschafter waren moderne und zentrale Vordenker in dieser Entwicklung. Und so wurde in einer dieser reformschwangeren und diskussionsfreudigen Einführungsvorlesung (Frage: "Wozu brauchen wir das alles!") die - wie sich später herausstellen sollte - schicksalhafte Frage gestellt (u.a. vom späteren Vorsitzenden der KPÖ), ob nicht Professoren Einkommensmaximierung betrieben, wenn sie Bücher (und Skripten) schrieben. Selten hatten wir Assistenten F. Ferschl derartig echauffiert, ja richtig betroffen gesehen. Er hätte ja vieles als Dekan in Bonn erleben müssen, aber den Vorwurf hier nahm er persönlich (obwohl dies nicht persönlich und wahrscheinlich gar nicht auf

\footnotetext{
${ }^{1}$ F. Ferschl, Deskriptive Statistik, Physica Verlag.
} 
ihn ad personam gemeint war). Dieser Auslöser und einigen weiteren Push- und PullFaktoren mehr führten F. Ferschl bald zum Entschluss, nach kurzen 3 Jahren von Wien nach München zu gehen, wo ihm u.a. die wohl einzigartige Kombination von Statistik und Philosophie reizte.

Wien war der Ort seines Mathematik-Studiums gewesen und so blieb er Wien auch in seiner Münchner Zeit treu verbunden, besonders durch seine Wohnung im 9. Bezirk. Auch das Grundlagenthema begleitete F. Ferschl bis zuletzt ins hohe Alter, wie aus einem letzten veröffentlichten Vortrag über ein religiöses Thema hervorgeht ${ }^{2}$. Wer mit F. Ferschl diskutiert hatte, erfuhr eine Art "magic touch", der das eigene Denken anregte und daher suchte man/frau immer wieder gerne das Gespräch mit ihm. Seine Art, jedem in der Diskussion als Spiegel der eigenen Worte zu dienen, hat F. Ferschl bis in die hohen Jahre eine breite Fangemeinde beschert. In einer akademischen Welt, wo bibliometrische Messlatten immer höher gelegt werden, werden Persönlichkeiten wie F. Ferschl, die pädagogische Reflexion vor mediengerechten Selbstinszenierung stellen, immer seltener werden.

Wien, im August 2007

Wolfgang Polasek

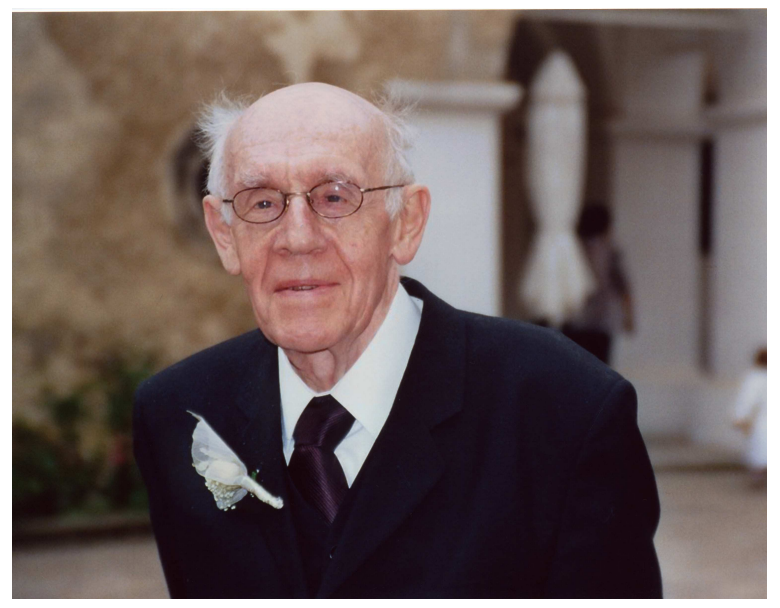

\footnotetext{
${ }^{2}$ F. Ferschl: Kriterien für den Dialog auf der "kognitiven" Ebene; in Peter F. Schmid, "Erkenntniswege in der Theologie", Graz (Styria) 1998.
} 